\title{
Automatic visual-spatial perspective taking in alcohol-dependence: a study with happy emotional faces
}

Sharon Cox ${ }^{a}$, Pierre Maurage ${ }^{b}$, Richard O'Connor ${ }^{c}$ Chris Chandler $^{d}$ \& Kevin Riggs ${ }^{c}$

a (Corresponding author) London South Bank University, Centre for Addictive Behaviours Research, School of Applied Sciences, 103 Borough Road, London, SE1 OAA, UK. coxs15@lsbu.ac.uk.

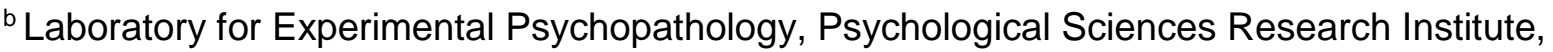
Université catholique de Louvain, Place C. Mercier 10, B-1348 Louvain-la-Neuve, Belgium c University of Hull, Department of Psychology, Cottingham Road, Hull, HU16 4RS, UK ¿London Metropolitan University, Department of Psychology, Tower Building, Holloway Road, London, NT 7DT, UK

(C) 2018. This manuscript version is made available under the CC-BY-NC-ND 4.0 license http:// creativecommons.org/licenses/by-nc-nd/4.0/ 


\begin{abstract}
Background: Understanding the world from another's perspective is an important and potentially automatic human process which is crucial for efficient social interactions. However, whilst deficits have been repeatedly described for various interpersonal abilities in alcoholdependence $(A D)$, only one previous study has investigated perspective taking in this pathology.
\end{abstract}

Aim: The aim of the current study was to explore further how AD affects visual-spatial perspective taking (VSPT) by examining the effect of positive emotional stimuli on VSPT in both an $A D$ and non-AD sample.

Methods: Reaction times (RT) for simple spatial judgements were measured. Participants made these judgements from their own perspective, but judgements were either congruent or incongruent with the perspective of another agent. The emotion conveyed by that agent (happy or neutral) was manipulated across trials.

Results: Compared to baseline, both $\mathrm{AD}$ and non-AD groups displayed delayed RTs for spatial judgements when these were incongruent with the perspective of a happy agent (the expected VSPT RT cost, indicating automatic VSPT). The AD, but not the non-AD group, further displayed a VSPT RT cost when the agent expressed a neutral emotion.

Conclusion: There was no evidence that automatic VSPT was compromised by AD. However, as in previous research, $A D$ was associated with differences in the processing of emotional stimuli. Future research should explore which 'real-world' settings are likely to trigger social confusion and misunderstanding.

Key words: Alcohol-dependence, social processing, perspective taking, emotion 


\section{Introduction}

Alcohol-dependence $(A D)$ is associated with a wide range of physiological and neurological problems (Bühler and Mann, 2011), having a particular impact on cognitive abilities (Stavro et al., 2013). The neurotoxic effects of alcohol in AD trigger a range of transient and long-term behavioural deficits, leading to cognitive and social issues, which may perpetuate excessive alcohol drinking (Oscar-Berman and Marinkovic, 2013).

$A D$ also has detrimental effects on socio-emotional abilities, particularly during the processing of faces, voices and words, leading to inaccurate assessments of the intensity of the emotion conveyed (Kornreich et al., 2013). Research has highlighted the problems which AD patients experience with emotional understanding (Bosco et al., 2014), Theory of Mind (Maurage et al., 2015), humour understanding (Uekermann et al., 2006), irony processing (Amenta et al., 2013) and social misdemeanours (faux pas; Thoma et al., 2013). Whilst these previous studies have clearly established that socio-emotional processing impairments are a key factor in $A D$, they have all focused on the conscious and deliberate processing of social information, as they were based on explicit social cognition tasks. However, during real-life interactions, the processing of social signals is most often automatic and unrelated to reflective evaluation. Furthermore, in other disorders different patterns of socio-emotional impairments have been reported depending on whether implicit or explicit tasks are used (e.g., ASD: Frith, 2004). This would suggest an important need for research into implicit socio-emotional processing in $A D$. Only one study has directly measured the effect of $A D$ on an automatic social cognition task - visual-spatial perspective taking (Cox et al., 2016).

Cox et al. (2016) used an automatic visual-spatial perspective taking (VSPT) measure, previously used with a range of healthy and pathological populations (Zwickel and Muller, 2010; Zwickel et al., 2011; Pearson et al., 2013). Participants responded from their own perspective to the location of a dot-probe that appeared to the left/right or above/below facial stimuli conveying neutral or fearful expressions. Automatic perspective taking was measured 
by the reaction time cost displayed when participants had to report a location of the dot that was incongruent with the perspective of the other agent (the face on the screen) i.e., left/right judgements. In previous studies with healthy participants, fearful, but not neutral, faces triggered automatic perspective taking, likely because the additional emotional content increases the salience of the other agents' perspective (Zwickel and Muller, 2010).

Cox et al. (2016) found both $A D$ and non-AD participants showed a visual-spatial perspective taking reaction time cost (VSPT RT cost) to the fearful faces. This suggests that while $A D$ leads to impaired high-level and explicit social processing, it is not related to generalized social cognition impairments, given that implicit processing of social stimuli appears preserved. This is a potentially important result at both the theoretical level (by showing a differential rather than ubiquitous impairment) and the clinical level (by identifying implicit processing as a potential lever to rehabilitate social cognition in AD). However, in that initial experiment, AD patients did show socio-emotional processing differences to controls: whereas non-AD participants showed a VSPT RT cost to only fearful faces, AD participants showed this cost to both neutral and fearful faces, with no significant difference between them.

Two possible hypotheses could explain the difference in responding across AD and non-AD participants: (i) AD participants specifically overestimate the negative emotional content of faces, leading to a conflation of neutral and fearful faces, or potentially a carry-over effect of negative emotional content from the fearful faces to the neutral ones; (ii) in the context of perspective-taking, AD participants display a generalized increased salience for all faces, irrespective of emotional content. There is some evidence to support a hyper-responsiveness towards reporting negative emotions of facial stimuli in AD (Foisy et al., 2007a; Foisy et al., 2007b; Philippot et al., 1999; Townshend and Duka, 2003: though see Donadon and de Lima Osorio, 2014). Given the potential for the Cox et al. (2016) data to have been predominantly triggered by over-estimated negative stimuli, the investigation of whether this effect is also preserved for positive stimuli (i.e., happy faces) is required before strong conclusions can be made about the nature of implicit social processing in AD. 
The present study therefore uses the same methodology as Cox et al. (2016) to measure automatic VSPT in AD and non-AD participants, but with happy instead of fearful faces. With regard to $A D$ and the processing of happiness, studies of labelling and intensity ratings (Clark et al., 2007; Kornreich et al., 2013; Maurage et al., 2009; Philippot et al., 1999) typically show a preservation of explicit happiness processing. To date, no known research has investigated the effect of happy faces on automatic VSPT in either clinical or non-clinical populations. Although happy faces do not engender the same social value as fearful ones in relation to potential cues for threat and harm, they nonetheless constitute highly relevant information for adapted social interactions.

In sum this study investigates whether happy faces trigger spontaneous VSPT in both $A D$ and non-AD groups, to clarify whether (1) automatic perspective taking to neutral stimuli in $A D$ is related to a specific tendency to overestimate negative stimuli, and (2) the preservation of implicit social processing (automatic perspective taking) in AD to fearful faces generalizes to positive stimuli. We predicted that happy faces would elicit automatic perspective taking in much the same way that fearful faces do in non-AD populations. Additionally, based on the findings from Cox et al. (2016), we predicted that AD participants would also engage in automatic perspective taking to happy faces.

\section{Method}

\subsection{Participants}

All participants volunteered to take part. The study was approved by London Metropolitan University (where the work was carried out) committee and conducted in accordance with the ethical standards of the 1964 Declaration of Helsinki. The same 22 nonAD and 22 AD participants who took part in the earlier version of this study (see Cox et al., 2016, for full details) were recruited a few weeks later. The groups did not differ significantly in age $(A D$ mean $=43 \pm 12$ years; non- $A D$ mean $=42 \pm 9$ years, $t(42)=0.18, p>0.05)$ or 
gender (both groups consisted of 11 men and 11 women). All participants were of British origin.

AD participants were all assessed according to the Diagnostic and Statistics ManualIV-R (American Psychiatric Association, 2000) criteria for alcohol dependence by their keyworker at the time of entering treatment. The treatment service centre participating in this study has an 'alcohol-free at the time of treatment' policy and therefore all AD participants were alcohol free at the time of testing, as assessed by their key-worker using a breathalyser test. The groups differed on the Fast Alcohol Screening Test (FAST), a simple four question audit to detect problem drinking (Hodgson et al., 2002): $A D, M=9.87$, (SD = 4.01); non-AD, $M=1.02,(S D=2.10)$. This difference was significant, $t(42)=6.67, p<0.001$. The mean of number of alcoholic units taken per day prior to treatment within the AD group was 14.21 (SD $=4.23)$ and mean number of years of problematic drinking was $16.10(\mathrm{SD}=8.74)$. The average number of attempts at detoxification was $1.50(S D=0.50)$.

None of the participants reported poly-drug use, psychiatric or neurological disease, as measured at the time of entering treatment by psychiatric assessment and past medical records. No AD participants were in withdrawal at the time of taking part, or currently on any medication relevant to aiding withdrawal symptoms (reporting 4+ weeks abstinence.

Daily and current smoking was more common in the group with $A D N=7$, compared to the non-AD group $N=4$. The Trait Anxiety Inventory for adults (STAI) was administered to control for the effects of anxiety (Spielberger et al., 1983). Anxiety scores were higher in the AD group, $M=41.23,(S D=8.37)$ as compared to the non-AD group, $M=36.91$, $(S D=11.28)$, but this difference was not significant $t(42)=-1.44, p>0.05$. AD and non-AD groups also differed on their scores for depression (Beck Depression Inventory; BDI; Beck and Steer, 1990). BDI scores were higher for participants with $A D, M=12.34(S D=8.12)$ than for the control group, $\mathrm{M}=9.87(\mathrm{SD}=6.54)$ and this difference was significant $\mathrm{t}(42)=-2.56, \mathrm{p}<$ 0.001. Although the participants with $A D$ presented higher and a greater range of depression scores, neither groups' average scores reached what is considered to be the clinical level of depression. 


\subsection{Apparatus and Stimuli}

Stimuli were presented on a Toshiba laptop with a 21 " computer screen $(85-\mathrm{Hz}$ refresh rate) positioned $50 \mathrm{~cm}$ in front of the participants (see Cox et al., 2016, for an example of the stimulus). Twelve male and 12 female grey-scaled faces with hair removed and presented within a rectangle were presented. The remainder of the screen was white. Twelve of the faces conveyed a happy expression and 12 a neutral expression. A black rectangle which was the same in size as the facial stimuli acted as a baseline stimulus.

\subsection{Design and Procedure}

Half of participants completed the BDI and STAI before the trials and half after. Smokers were not asked to abstain before testing periods to maintain relative ecological validity and because nicotine withdrawal may negatively impact on testing (mood, irritability), as well as being a disincentive for some participants taking part. Participants were instructed to complete a set of 10 practice trials before starting the recorded experiment. Test trials were pre-randomised into blocks of 12 , consisting of faces incongruent; faces congruent; baseline (rectangles) incongruent; baseline congruent. Within each block of 12 - with the exception of the baseline trials -6 faces conveyed fearful and 6 neutral expressions. Instructions were given before each block of trials to remind participants to respond to the location of the dot. There were a total of 144 trials. Trials started with presentation of the stimuli, and was followed $500 \mathrm{~ms}$ later by a dot probe, $5^{\circ}$ in diameter, that appeared for $35 \mathrm{~ms}$. The dot then disappeared leaving the face on screen until a response was given (see Fig. 1). Reaction time was recorded from the onset of the dot probe. For the incongruent trials the dot appeared 10 to the left or right of the face/rectangle, and for the congruent trials $1 \circ$ above or below the face/rectangle. During the baseline trials the dot also appeared for the same duration and in the same locations but the stimulus was a black rectangle. Participants were asked to respond as quickly and as accurately as possible, pressing ' $s$ ' to indicate left, and ' $k$ ' for dots on the right, ' $t$ ' for those at the top, and ' $b$ ' for the bottom. 
Figure 1 here

\section{Results}

\subsection{Analysis of the test conditions}

Analysis of covariance and correlation analysis showed that neither smoking, anxiety and depression had no significant effect on the RT for either group across conditions. Raw RT data is presented in Fig. 1. ANOVA main effects confirmed earlier findings: ADs responded slower than non-ADs $F(1,42)=31.07, p<.001, R^{2}=.42$; responses to incongruent trials were slower than congruent ones $F(1,42)=6.20, p=.017, R^{2}=.129$; and responses to faces were slower compared to baseline conditions $F(2,84)=67.75, p<.001, R^{2}=.617$.

Figure 2 here

Perspective taking was measured by calculating a difference score between the congruent and incongruent conditions (Fig. 2). Difference scores were analyzed in a 2-way mixed ANOVA with stimulus type as the within-participants factors and group as the betweenparticipant factor. There was a main effect for stimulus type, $F(2,84)=51.43, p<.001, R^{2}=.550$, and for group, $F(2,42)=17.37, p<.001, R^{2}=.293$. There was also a significant interaction between stimulus and group, $F(2,84)=6.21, p=.005, R^{2}=.129$. In the non-AD group there was a significant perspective effect for happy faces $t(21)=5.92, p<.001(\mathrm{Cl} 149.95--75.50)$ but not with neutral faces. AD participants showed a perspective effect for both happy $t(21)=$ 7.50, p<.001(Cl -308.45- -174.46) and neutral faces $t(21)=6.11, p<.001(\mathrm{Cl}-177.76--87.51)$. Furthermore, the AD group showed an increased perspective taking effect for happy faces compared to neutral $t(21)=6.90, p<.001(\mathrm{Cl}-133.21--53.78)$. 
Finally, in view of the high variance of RTs within our AD group we further explored whether VSPT was preserved beyond a group effect and thus conducted an adapted singlecase versus group analysis (Crawford and Howell, 1998); all AD participants except one individual (who demonstrated an equal RT cost to both happy and neutral faces) demonstrated the same response pattern.

\section{Discussion}

A primary aim of this study was to investigate whether preservation of implicit social processing observed in our earlier work with fearful faces (Cox et al., 2016) extends to positive stimuli (happy faces). This was demonstrated: participants showed a VSPT RT cost to happy faces when reporting a spatial location from their own perspective that was incongruent to that from the perspective of another agent. Hence, our results show that observing happy faces elicit spontaneous VSPT in both AD and non-AD observers.

Interestingly, there was an important point of difference with our earlier study. In our earlier study, non-AD participants showed a VSPT RT cost to fearful faces only, not to neutral faces. The AD group showed VSPT RT costs to both fearful and neutral faces, with the cost being similar to both. In the current study, the non-AD group showed a VSPT cost to happy faces, but not to neutral faces. The AD group showed a VSPT RT cost to both happy and neutral faces, although there was an additional cost to happy compared to neutral faces. In other words there was a similar pattern of responding to positive stimuli across the non-AD and $A D$ groups. This is broadly consistent with previous research using emotional faces, voices and music showing that when presented with positive stimuli patients with $A D$ show relatively similar responses to controls (Maurage et al., 2009; Kornreich et al., 2013). Further 
research is needed to understand the differential deficit for negative but preserved positive emotion processing.

There are several limitations to this study. While our previous work (Cox et al., 2016) and the current study provide a snap shot of behaviour, due to restrictions on client data from the treatment service centre, the complexity of the participants current mental and physical health lacks detail. Future studies should include more detail of the clinical background of the participants in order to set the current findings against a wider clinical picture. Furthermore, Kornreich et al., (2001) have shown that emotional facial expression deficits correlate negatively with interpersonal issues, thus future studies replicating these findings should seek to clarify the association between perceived interpersonal difficulties and perspective taking, and more globally the real-life impact of the deficits observed here on patients' social life and interpersonal relations.

In summary, across both of our studies we find no evidence to suggest that $A D$ participants are impaired in automatic VSPT. The relevance of the results together is that while AD may have a deleterious impact on explicit emotional processing they do not support the view that $A D$ causes a blanket or generalised social processing deficit. Whatever the explanation for the difference between implicit and explicit emotion processing, our findings suggest that the large number of documented deficits in explicit social cognition do not extend to certain implicit tasks. Further research is needed to identify intact implicit socio-emotional processes, given their potential in therapeutic settings to rehabilitate social cognition in AD. 


\section{References}

Amenta, S., Noël, X., Verbanck, P., Campanella, S., 2013. Decoding of Emotional Components in Complex Communicative Situations (Irony) and Its Relation to Empathic Abilities in Male Chronic Alcoholics: An Issue for Treatment. Alcohol. Clin. Exp. Res. 37, 339-347. doi:10.1111/j.1530-0277.2012.01909.x

American Psychiatric Association, 2000. DSM-IV-TR, Diagnostic and Statistical Manual of Mental Disorders 4th Edition TR. APA, Washington.

Beck, Aaron T., Steer, Robert A., 1990. Manual for the Beck Anxiety Inventory. Psychological Corporation, San Antonio, TX.

Bosco, F.M., Capozzi, F., Colle, L., Marostica, P., Tirassa, M., 2014. Theory of mind deficit in subjects with alcohol use disorder: An analysis of mindreading processes. Alcohol Alcohol. 49, 299-307. doi:10.1093/alcalc/agt148

Bühler M., Mann K., 2011. Alcohol and the human brain: a systematic review of different neuroimaging methods. Alcohol. Clin. Exp. Res. 1;35(10):1771-93.

Clark, U.S., Oscar-Berman, M., Shagrin, B., Pencina, M., 2007. Alcoholism and judgments of affective stimuli. Neuropsychology 21, 346-62

Cox S., Chandler C., Simpson A., Riggs K., 2016. The effect of alcohol dependence on automatic visuo-spatial perspective taking. Drug and alcoh dep. 1;166:21-5

Crawford, J.R., Howell, D.C., 1998. Comparing an individual's test score against norms derived from small samples. The Clinical Neuropsychologist, 12(4), pp.482-486.

Donadon, M. F., de Lima Osório, F. 2014. Recognition of facial expressions by alcoholic patients: a systematic literature review. Neuropsychiatric disease and treatment, 10, 1655-1663.

Foisy, M.L., Kornreich, C., Petiau, C., Parez, A., Hanak, C., Verbanck, P., Pelc, I. Philippot, P. 2007. Impaired emotional facial expression recognition in alcoholics: are these deficits specific to emotional cues?. Psychiatry research, 150(1), 33-41. 
Foisy, M.L., Kornreich, C., Fobe, A., D'hondt, L., Pelc, I., Hanak, C., Verbanck, P., Philippot, P. 2007. Impaired emotional facial expression recognition in alcohol dependence: do these deficits persist with midterm abstinence?. Alcoholism: Clinical and Experimental Research, 31(3), 404-410.

Frith, U. 2004. Emanuel Miller lecture: Confusions and controversies about Asperger syndrome. Journal of child psychology and psychiatry, 45(4), 672-686.

Kornreich, C., Brevers, D., Canivet, D., Ermer, E., Naranjo, C., Constant, E., Verbanck, P., Campanella, S., Noël, X., 2013. Impaired processing of emotion in music, faces and voices supports a generalized emotional decoding deficit in alcoholism. Addiction 108. doi:10.1111/j.1360-0443.2012.03995.x

Kornreich, C., Philippot, P., Foisy, M.L., Blairy, S., Raynaud, E., Dan, B., Hess, U., Noël, X., Pelc, I. Verbanck, P., 2002. Impaired emotional facial expression recognition is associated with interpersonal problems in alcoholism. Alcohol and alcoholism, 37, 394-400.

Maurage, F., de Timary, P., Tecco, J.M., Lechantre, S., Samson, D., 2015. Theory of Mind Difficulties in Patients with Alcohol Dependence: Beyond the Prefrontal Cortex Dysfunction Hypothesis. Alcohol. Clin. Exp. Res. 39, 980-988. doi:10.1111/acer.12717

Oscar-Berman, M., Marinkovic, K., 2003. Alcoholism and the brain: an overview. Alcohol Res. Health 27, 125-133

Pearson, A., Ropar, D., de C Hamilton, A.F., 2013. A review of visual perspective taking in autism spectrum disorder. Front. Hum. Neurosci. 7, 652. doi:10.3389/fnhum.2013.00652 
Philippot, P., Kornreich, C., Blairy, S., Baert, I., Den Dulk, a, Le Bon, O., Streel, E., Hess, U., Pelc, I., Verbanck, P., 1999. Alcoholics' deficits in the decoding of emotional facial expression. Alcohol. Clin. Exp. Res. 23, 1031-1038. doi:00000374-19990600000010 [pii]

Spielberger, C.D., Gorsuch, R.L., Lushene, P.R., Vagg, P.R., Jacobs, A.G., 1983. Manual for the State-Trait Anxiety Inventory (Form Y), Manual for the statetrait anxiety inventory STAI

Stavro, K., Pelletier, J. and Potvin, S., 2013. Widespread and sustained cognitive deficits in alcoholism: a meta- analysis. Addiction biology. 18,2, 203-213.

Thoma, P., Winter, N., Juckel, G., \& Roser, P., 2013. Mental state decoding and mental state reasoning in recently detoxified alcohol-dependent individuals. Psychiat Res, 205(3), 232- 240.

Townshend, J. M., Duka, T. 2003. Mixed emotions: alcoholics' impairments in the recognition of specific emotional facial expressions. Neuropsychologia, 41(7), 773782.

Uekermann, J., Channon, S., Winkel, K., Schlebusch, P., Daum, I., 2007. Theory of mind, humour processing and executive functioning in alcoholism. Addiction. 102, 232-240. doi:10.1111/j.1360-0443.2006.01656.x

Zwickel, J., Müller, H.J., 2010. Observing fearful faces leads to visuo-spatial perspective taking. Cognition 117, 101-105. doi:10.1016/j.cognition.2010.07.004 
Zwickel, J., White, S. J., Coniston, D., Senju, A., Frith, U. 2010. Exploring the building blocks of social cognition: spontaneous agency perception and visual perspective taking in autism. Social Cognitive and Affective Neuroscience, 6(5), 564-571. 


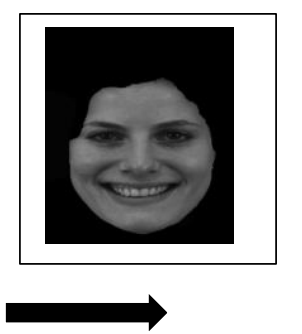

$500 \mathrm{~ms}$

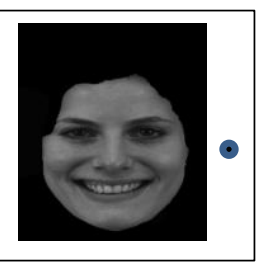

$35 \mathrm{~ms}$

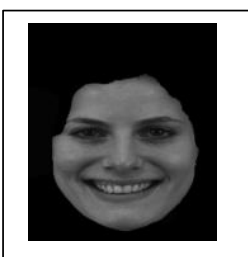

$\longrightarrow$ On screen until response given

Figure 1. Schematic of the Experiment 


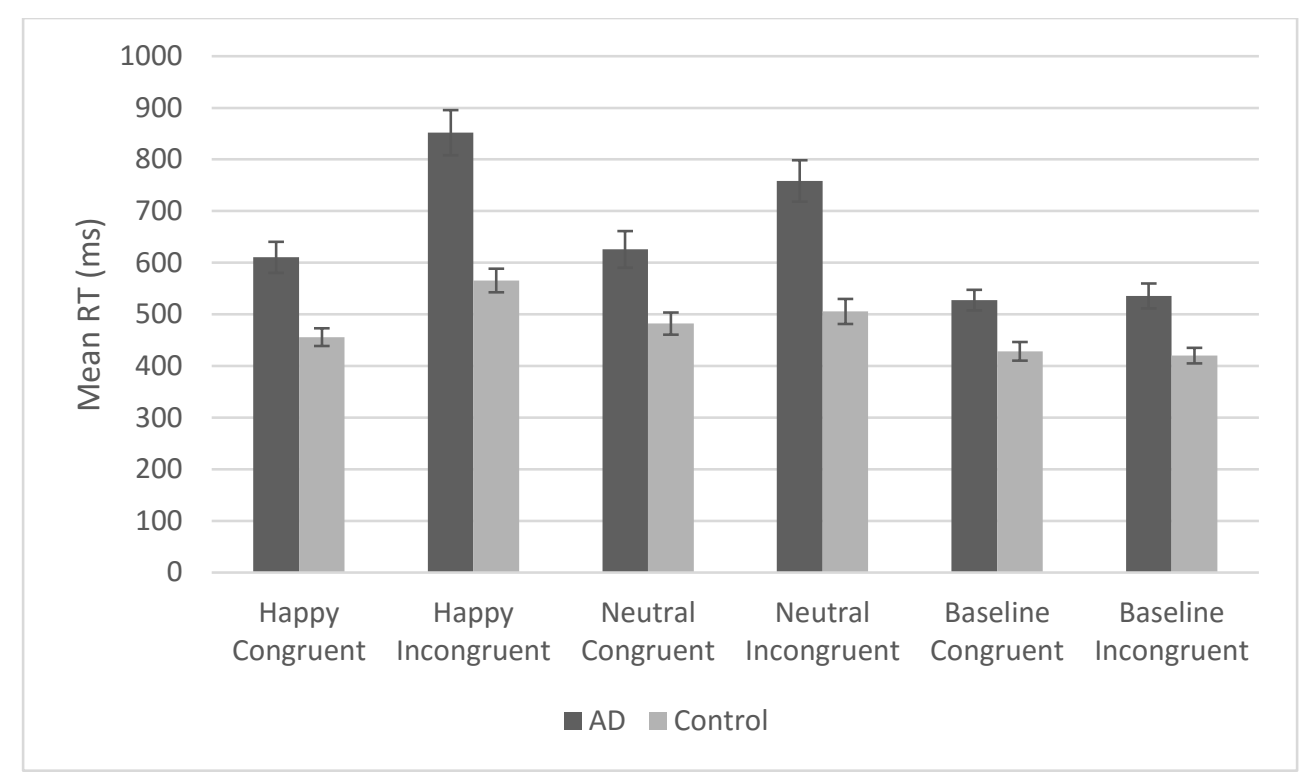

Figure 2: Mean RTs (ms) to happy, neutral and baseline stimuli by perspective for participants with $A D$ and the control (non- $A D$ ) participants. Bars represent the standard error. 
Alcohol dependence and perspective taking

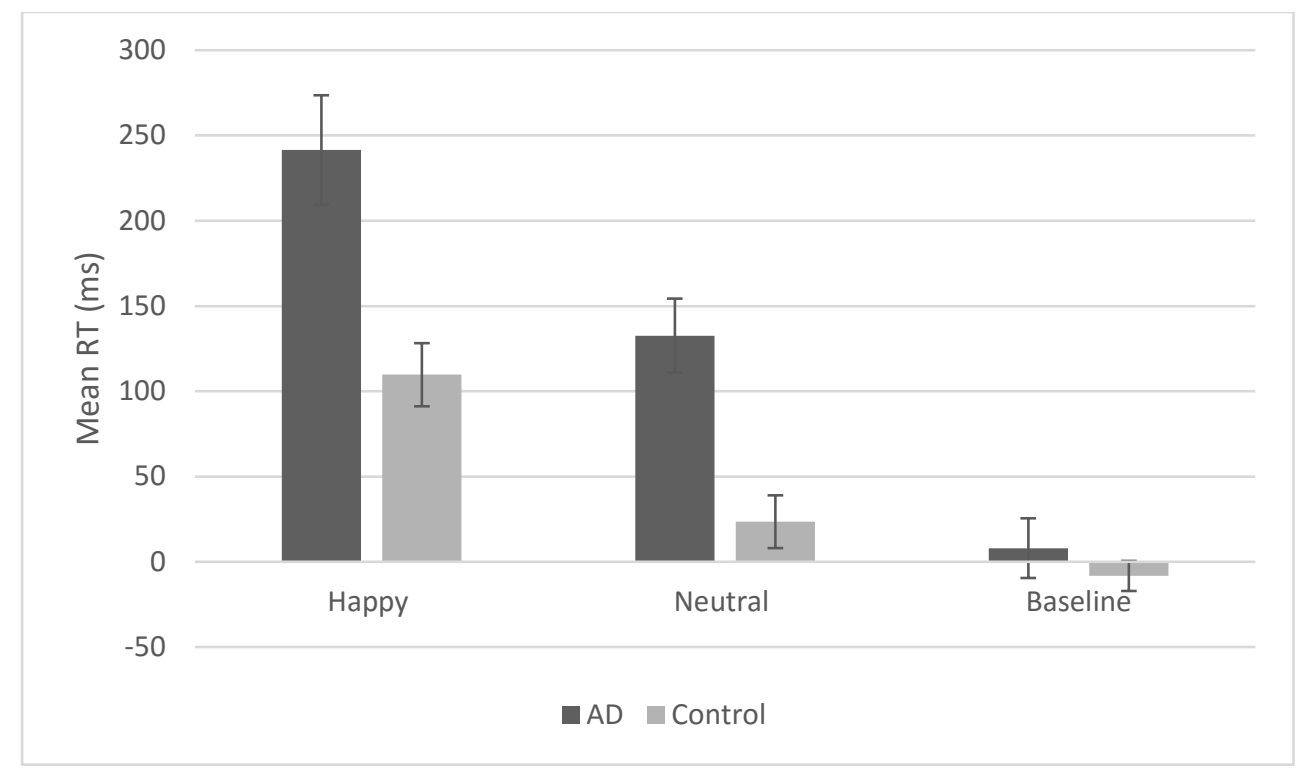

Figure 3: Mean RTs (ms) as calculated by the difference score for happy, neutral and baseline stimuli for participants with $A D$ and the control (non-AD) participants. Bars represent the standard error. 